\title{
Comparison of Molecular Docking and Molecular Dynamics Simulations of 1,3-thiazin-4-one with MDM2 Protein
}

\author{
S. Athavan Alias Anand, C. Loganathan, K. Saravanan and S. Kabilan* \\ Drug Discovery Lab, Department of Chemistry, Annamalai University, Annamalainagar - 608002 , \\ Tamil Nadu, India.
}

Corresponding author e-mail: profskabilanau@gmail.com

Keywords: Docking, dynamic Simulations, Thiazinone, MDM2

\begin{abstract}
The molecular docking and molecular dynamics simulations studies of 1,3-thiazin4-one derivative with a bonafide oncogene protein MDM2 (PDB ID: 4HBM) was investigated. Both the docking and dynamics simulations were performed in Schrödinger software suite 2014 using Glide and Desmond modules. The results of docking and dynamics were compared to investigate the possible binding modes of the thiazinone derivative with 4HBM. The tested molecule shows critical interactions with the important amino acid His 96 which is necessary for the inhibition of MDM2 in both docking and dynamic studies.
\end{abstract}

\section{INTRODUCTION}

The p53 is a protein act as a natural tumor suppressor in humans and it is present in low concentration in normal cells. The p53 was also act as transcription factor which stimulate various genes to perform their specific functions. The loss of p53 in cancer in vivo studies shows the importance of targeting p53 for anticancer therapy [1]. Recently the MDM2 (Murine Double Minute 2) protein was identified as a negative regulator of p53 and it's over expression causes various cancers in humans. The MDM2 arrest the significant activity of p53 by its interaction leads to uncontrolled cell proliferation. It was observed that the inhibition of p53-MDM2 interaction reduces the growth of cancer cells, which demonstrates the noteworthy activity of p53 [2]. To avoid the p53-MDM2 interactions, the active site of the MDM2 was inhibited by a small molecule. In the present work, a small molecule with thiazinone moiety was tested theoretically for its fitness and binding properties in the active site of MDM2 protein. The synthetic method and the anticancer studies of the tested molecule was reported in our previous paper [3].

Recently 2-piperidone derivative was reported as potent and selective inhibitors of the MDM2-p53 interaction. On the other hand, thiazinone was also considered as an important analogue in the medicinal chemistry. Thiazinone was associated with diverse biological properties such as anticonvulsant [4], antifungal [5], antimalarial [6] and anticancer [7]. A thiazinone derivative YM928 (2-[N-(4-chlorophenyl)-N-methylamino]-4H-pyrido[3,2-e]-1,3-thiazin-4-one; mol. wt: $303 \mathrm{Da})$ is an orally active non-competitive antagonists for $\alpha$-Amino-3-hydroxy -5,5-methyl-4-isoxazolepropionic acid (AMPA) receptor, which plays an important role in the normal functions of brain and in the pathogenesis of several neurological disorders [8].

Molecular docking and dynamics simulations studies were carried out to investigate the active site interactions between the 1,3-thiazin-4-one molecule (ligand) and the MDM2 protein (receptor). In molecular docking, the selected receptor considered as a rigid macromolecule whereas the ligand is flexible. All the plausible conformers of the ligand were generated and each conformer was tested for its fitness with the binding pocket of the receptor. The factors such as bond length, bond angle, dihedral angle, electrostatic forces, van der Waals forces, hydrogen bonding, hydrophobicity, steric hindrances etc., were affect the molecular docking. In molecular dynamics simulation, the interaction and the stability of the protein-ligand complex were calculated in motion. Initially, the protein- ligand complex was created manually and immersed in the orthorhombic box with calculated volume of solvent system. Later, the simulations were performed for few nano seconds in constant temperature and pressure. The results of MD simulations were 
compared with molecular docking studies to examine the mode of interactions when the protein was rigid and flexible.

\section{MATERIALS AND METHODS}

\subsection{Molecular Docking}

The binding affinities between the thiazinone derivative and MDM2 protein were studied in molecular docking studies. The 3D co-ordinates of crystallographic structures of MDM2 [9] (PDB ID: 4HBM) was downloaded from Brookheaven protein Data Bank (www.rscb.com). The protein complex was pre-processed and prepared by Protein Preparation Wizard [10] in Maestro [11] of Schrödinger software. The minimization of the complex was continued using OPLS-2005 (Optimized Potential for Liquid Simulations) force field [12] until the root mean square deviation (RMSD) reached the value of $0.3 \AA$.

The 2D structure of thiazinone was imported from the workspace of Maestro and the structure was minimized and geometrically refined using Ligprep [13] module. Conformers were generated using torsional search method with distance dependent dielectric solvation treatment and OPLS-2005 force field. The extra precision (XP) mode of docking was used to find the best fit molecules in the active sites of docked proteins using Glide [14] application of Schrödinger software suite 2014.

\subsection{Molecular Dynamic Simulations}

Molecular Dynamic (MD) stimulations studies were carried out by Desmond [15] module of Schrödinger software. The docked conformer of thiazinone with highest G-score was selected for the MD simulations study with OPLS 2005 force field. The protein-ligand complex was bounded with a predefined TIP3P water model [16] in orthorhombic box. The volume of the box was minimized and the overall charge of the system was neutralized by adding $\mathrm{Na}+$ and $\mathrm{Cl}-$ ions. The temperature and pressure were kept constant at $300 \mathrm{~K}$ and 1.01325 bar using Nose-Hoover thermostat [17] and Martyna-Tobias-Klein barostat [18] methods. The simulations were performed using NPT ensemble by considering number of atoms, pressure and timescale. During simulations, the long-range electrostatic interactions were calculated using Particle-Mesh-Ewald method [19].

\section{RESULTS AND DISCUSSION}

\subsection{Molecular Docking}

The binding affinity between the synthesized molecule and MDM2 protein (4HBM) was investigated by in silico molecular docking studies. The two dimensional structure of the thiazinone was drawn in 2D sketcher and imported in the workspace of Maestro. About 24 conformers were created by Ligprep module of Schrödinger suite 2014. The 2D structure and the 24 merged conformers of the thiazinone molecule are shown in Fig. 1. All the conformers were subjected to docking with the active site of 4HBM. The docking results of top ten conformers of thiazinone were tabulated in Table 1. The 2D and 3D docking poses of the best conformer were shown in Fig. 2. The result revealed that the thiazinone displayed important interaction with His 96 which is essential for the inhibition of MDM2 [20]. 


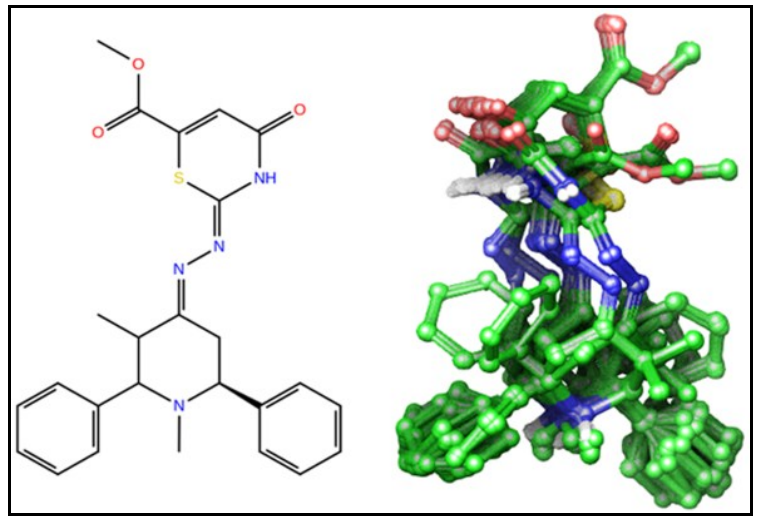

Figure 1. The chemical structure of thiazinone compound and its 24 merged conformers.

Table 1. Molecular docking results of top ten conformers of thiazinone compound

\begin{tabular}{ccccc}
\hline $\begin{array}{c}\text { Thiazinone } \\
\text { Conformers }\end{array}$ & $\begin{array}{c}\text { glide } \\
\text { gscore }\end{array}$ & $\begin{array}{c}\text { glide } \\
\boldsymbol{e v d}^{\mathrm{a}}\end{array}$ & $\begin{array}{c}\text { glide } \\
\text { ecoul }^{\mathrm{b}}\end{array}$ & $\begin{array}{c}\text { glide } \\
\text { energy } \\
\text { [kcal/mol] }\end{array}$ \\
\hline Conf. 1 & -7.623 & -33.61 & -5.182 & -38.792 \\
Conf. 2 & -7.094 & -27.844 & -8.792 & -36.636 \\
Conf. 3 & -7.025 & -38.612 & -1.232 & -39.844 \\
Conf. 4 & -7.013 & -27.351 & -8.633 & -35.984 \\
Conf. 5 & -6.701 & -29.892 & -4.272 & -34.164 \\
Conf. 6 & -6.674 & -39.632 & -4.361 & -43.993 \\
Conf. 7 & -7.416 & -31.21 & -4.015 & -35.225 \\
Conf. 8 & -7.230 & -26.89 & -4.221 & -31.112 \\
Conf. 9 & -6.083 & -41.432 & 0.324 & -41.108 \\
\hline Conf. 10 & -6.777 & -29.332 & -3.4 & -32.732 \\
\hline van der Waals interaction energies, ${ }^{\mathrm{b}}$ Coulomb interaction \\
\hline
\end{tabular}




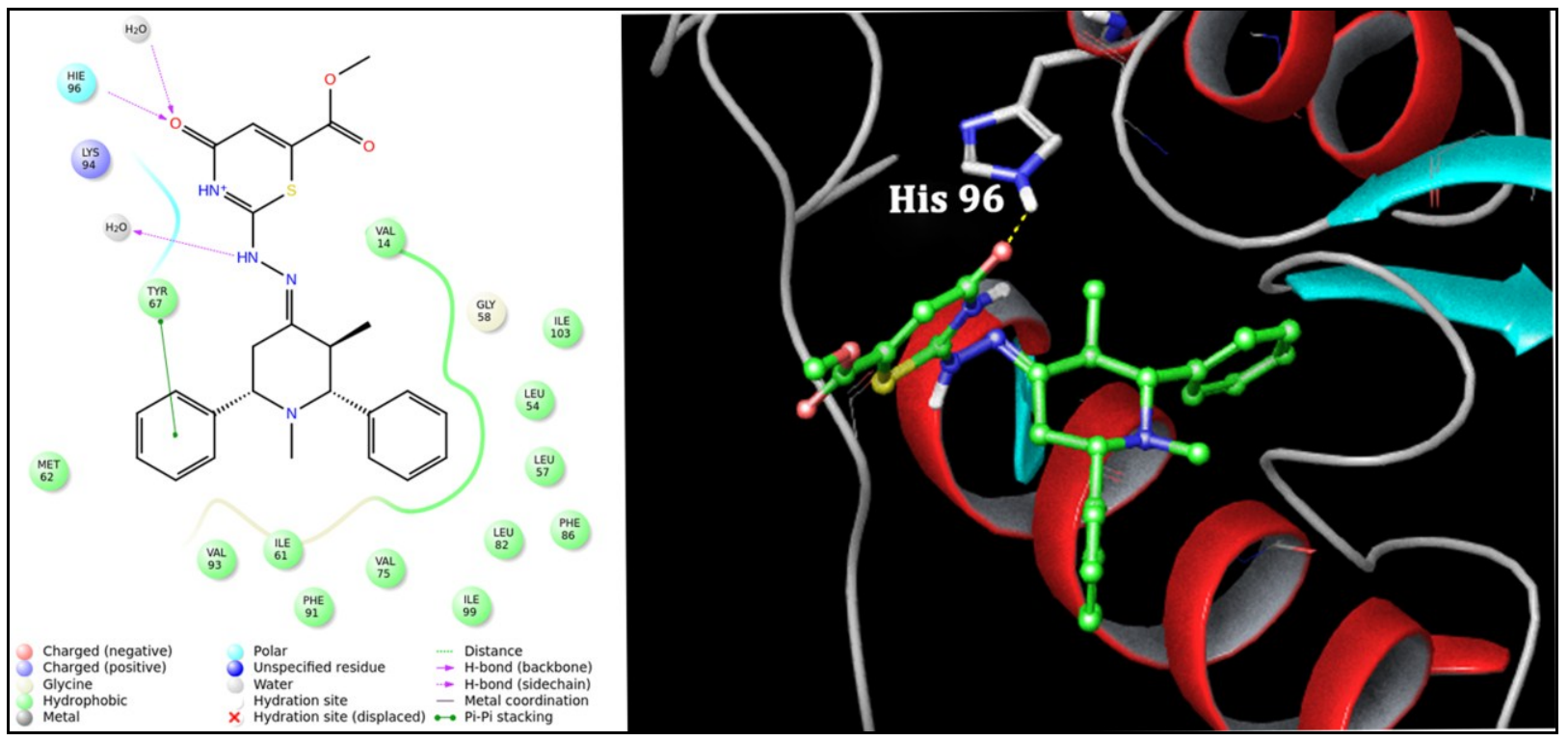

Figure 2. The 2D and 3D interaction poses of the thiazinone molecule in molecular docking.

The carbonyl oxygen of the thiazinone ring interacted with His 96 through hydrogen bond formation. The aromatic ring located in the $6^{\text {th }}$ position of piperidyl ring shows $\pi-\pi$ stacking interaction with TYR 67 amino acid residues. The water molecules present in the active site were also showing H-bond interactions with the title compound. The docking scores of first ten conformers were varies from -7.6 to -6.0 and the glide energy ranges from -43.9 to $-31.1 \mathrm{kcal} / \mathrm{mol}$. The evdw and ecoul are the predicted van der Waals interaction and electrostatic interaction energies between the ligand and the protein.

\subsection{Molecular Dynamic Simulations}

The thiazinone-4HBM complex was immersed in the orthorhombic box with TIP3P water solvent model for simulations using OPLS 2005 force field. After solvent system created, the simulations were equilibrated for 5 nano seconds by Molecular Dynamics application. The solvent system model with the prepared complex is shown in Fig. 3. The quality analysis of the simulations performed was depicted in Table 2.

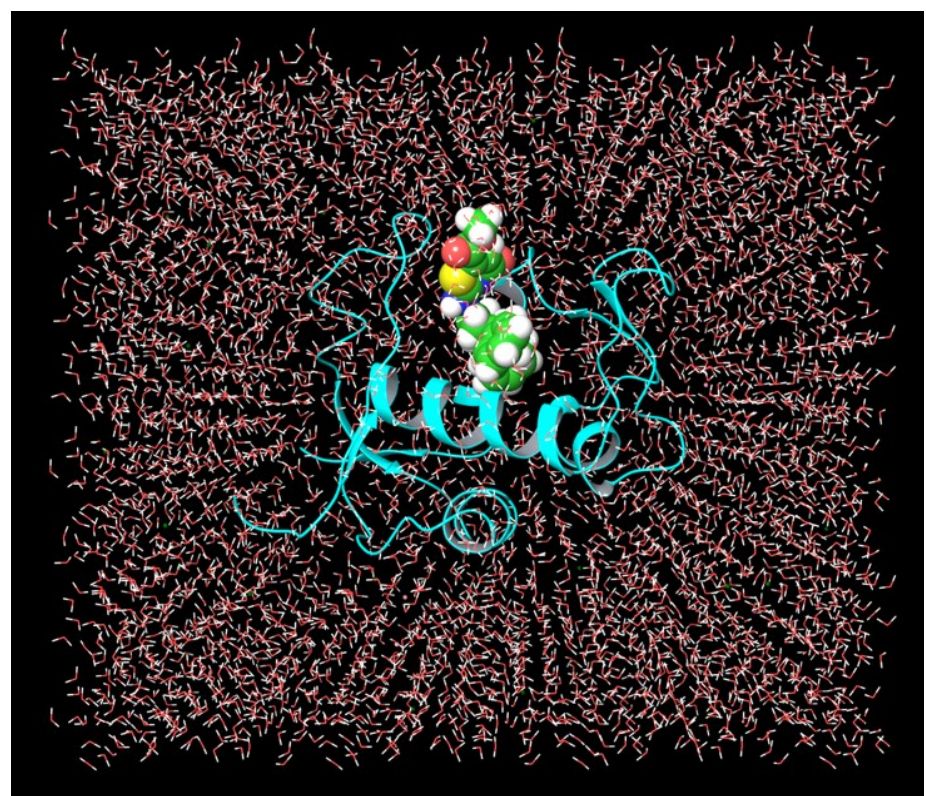

Figure 3. The water solvent model system made for molecular dynamics simulations. 
Table 2. Summary of simulation quality analysis after equilibration.

\begin{tabular}{cll}
\hline S. No. & \multicolumn{1}{c}{ Properties } & \multicolumn{1}{c}{$\begin{array}{c}\text { Statistical } \\
\text { parameters }\end{array}$} \\
\hline 1 & Simulation period $[\mathrm{ns}]$ & 4.998996 \\
2 & Degrees of freedom & 34123 \\
3 & Number of Atoms & 16605 \\
4 & Average total energy $[\mathrm{kcal} / \mathrm{mol}]$ & 44199.046 \\
5 & Average potential energy $[\mathrm{kcal} / \mathrm{mol}]$ & 54518.371 \\
6 & Temperature $[\mathrm{K}]$ & 298.613 \\
7 & Pressure $[\mathrm{bar}]$ & 14.244 \\
8 & Volume $\left[\AA^{3}\right]$ & 56247.958 \\
\hline
\end{tabular}

The RMSD (root mean square deviation) plot in Fig. 4 indicated that the thiazinone-4HBM complex reached its stable form at 3 ns and later it loses its stability after 4 ns. The result shows the complex may be attained its stability after $5 \mathrm{~ns}$. The residue interactions were similar to docking results. The nitrogen atom of thiazinone ring displayed water bridged interaction with two amino acids His 96 with $31 \%$ and Val 93 with $35 \%$ respectively. The $\pi-\pi$ stacking interaction was also observed with TYR 67 (41\%) in the binding pocket of 4HBM. The 2D interaction poses and the histogram chart are shown in Fig. 5. The histogram displayed favored hydrophobic interactions with ILE 61, TYR 67, ILE 99 and water bridged interactions with His 96, Val 93.

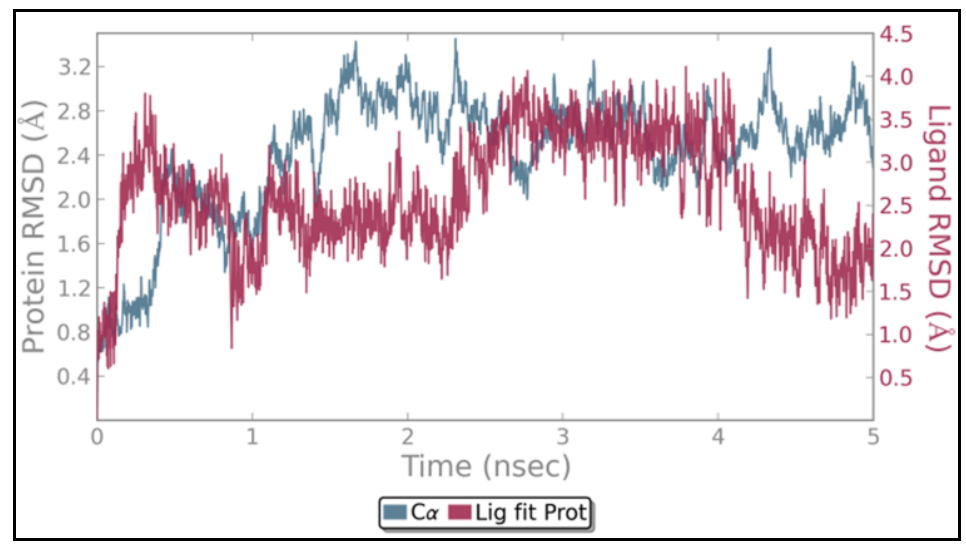

Figure 4. The RMSD plot obtained for thiazinone-4HBM complex. 


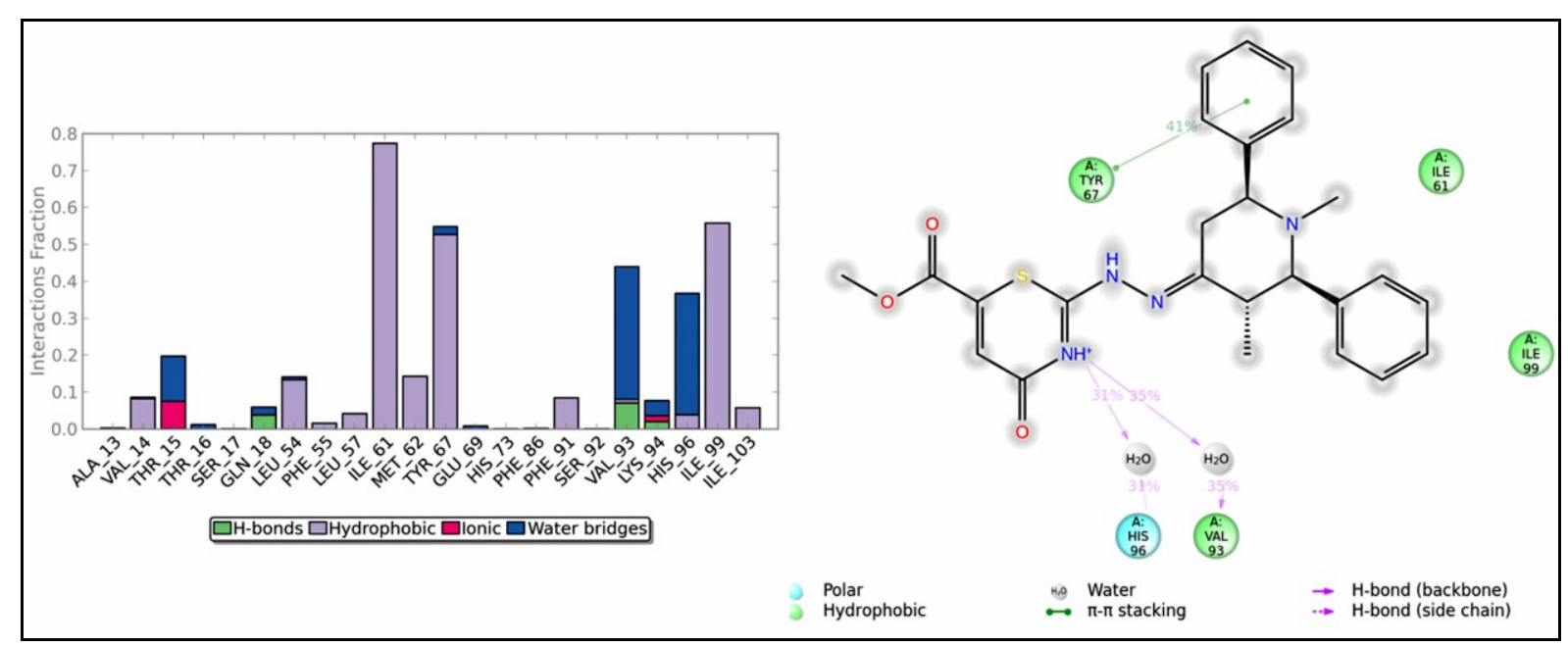

Figure 5. The histogram chart and the percentage of interactions in molecular dynamic simulations.

\section{CONCLUSION}

In silico molecular docking and dynamics simulations were employed for 1,3-thiazin-4-one and MDM2 protein (PDB ID: 4HBM). The results obtained were compared to investigate the possible interactions between the tested compound and 4HBM. The significant interaction with residue His 96 and TYR 67 was observed in both molecular docking and molecular dynamics simulations studies. By confirm the binding affinities of the ligand and the accurate interactions, molecular dynamics simulations valid the results of molecular docking.

\section{References}

[1] L.A. Donehower, M. Harvey, B.L. Slagle, M.J. McArthur, C.A. Montgomery, J.S. Butel, A. Bradley, L.A. Donehower, M. Harvey, B.L. Slagle, M.J. McArthur, C.A. Montgomery, J.S. Butel, A. Bradley, Mice deficient for p53 are developmentally normal but susceptible to spontaneous tumours, Nature, 356 (1992) 215-221.

[2] D. Bernard, Y. Zhao, S. Wang, AM-8553: a novel MDM2 inhibitor with a promising outlook for potential clinical development, J. Med. Chem. 55 (2012) 4934-4935.

[3] S.A.A. Anand, C. Loganathan, N.S. Thomas, K. Saravanan, A.T. Alphonsa, S. Kabilan, Synthesis, structure prediction, pharmacokinetic properties, molecular docking and antitumor activities of some novel thiazinone derivatives, New J. Chem. 39 (2015) 7120-7129.

[4] H. Yamashita, K. Ohno, H. Inami, J. Shishikura, S. Sakamoto, M. Okada, T. Yamaguchi, Suppression of fully kindled seizure and retardation of kindling acquisition by YM928 in the rat kindling model of epilepsy, Eur. J. Pharmacol. 494 (2004) 147-154.

[5] L.D.S. Yadav, A.R. Misra, H. Singh, Ring transformation of Michael adducts of Benzylidene-5-oxazolones and 3-mercapto-s-triazoles to 2,3-dihydro-4H-s-triazolo[3,4b] $[1,3]$ thiazin-4-ones with some antifungal activity, J. Agric. Food Chem. 36 (1988) 633-638.

[6] V.V. Kouznetsov, A.G. Barrio, Recent developments in the design and synthesis of hybrid molecules based on aminoquinoline ring and their antiplasmodial evaluation, Eur. J. Med. Chem. 44 (2009) 3091-3113.

[7] J. Matysiak, M. Juszczak, M.M. Karpińska, E. Langner, K. Walczak, M.K. Lemieszek, A. Skrzypek, A. Niewiadomy, W. Rzeski, Synthesis of 2-(2,4-dihydroxyphenyl)thieno-1,3-thiazin4-ones, their lipophilicity and anticancer activity in vitro, Mol. divers. (2015) DOI: 10.1007/s11030-015-9599-x. 
[8] K. Ohno, R. Tsutsumi, N. Matsumoto, H. Yamashita, Y. Amada, J. Shishikura, H.I.S. Yatsugi, M. Okada, S. Sakamoto and T. Yamaguchi, Functional characterization of YM928, a novel moncompetitive alpha-amino-3-hydroxy-5-methyl-4-isoxazolepropionic acid (AMPA) receptor antagonist, J. Pharmacol. Exp. Ther. 306 (2003) 66-72.

[9] K. Michelsen, J.B. Jordan, J. Lewis , A.M. Long, E. Yang, Y. Rew, J. Zhou, P. Yakowec, P.D. Schnier, X. Huang, L. Poppe, Ordering of the N-terminus of human MDM2 by small molecule inhibitors, J. Am. Chem. Soc. 134 (2012) 17059-17067.

[10] Protein Preparation Wizard; Epik version 2.3, 2014; Impact version 5.7, 2014, Schrödinger, LLC, New York.

[11] Maestro, 2014 release, Schrödinger suite, LLC, New York, 2014.

[12] W.L. Jorgensen, D.S. Maxwell, J. Tirado-Rives, Development and testing of the OPLS allatom force field on conformational energetics and properties of organic liquids, J. Am. Chem. Soc. 118 (1996) 11225-11236.

[13] LigPrep, version 2.5, 2014, Schrödinger, LLC, New York.

[14] Glide, version 5.8, 2014, Schrödinger, LLC, New York.

[15] Desmond Molecular Dynamics System, version 3.1, 2012, D. E. Shaw Research, New York, Maestro-Desmond Interoperability Tools, version 3.1, 2012, Schrödinger, New York.

[16] W.L. Jorgensen, J. Chandrasekhar, J.D. Madura, R.W. Impey, M.L. Klein, Comparison of simple potential functions for simulating liquid water, J. Chem. Phys. 79 (1983) 926-935.

[17] W.G. Hoover, Canonical dynamics: Equilibrium phase-space distributions, Phys. Rev. A. 31 (1985) 1695-1697.

[18] G.J. Martyna, D.J. Tobias, M.L. Klein, Constant pressure molecular dynamics algorithms, J. Chem. Phys. 101 (1994) 4177-4189.

[19] U. Essmann, L. Perera, M.L. Berkowitz, T. Darden, H. Lee, L.G. Pedersen, A smooth particle mesh Ewald method, J. Chem. Phys. 103 (1995) 8577-8859.

[20] F. Gonzalez-Lopez de Turiso, D. Sun, Y. Rew, M.D. Bartberger, H.P. Beck, J. Canon, A. Chen, D. Chow, T.L. Correll, X. Huang, L.D. Julian, F. Kayser, M.C. Lo, A.M. Long, D. McMinn, J.D. Oliner, T. Osgood, J.P. Powers, A.Y. Saiki, S. Schneider, P. Shaffer, S.H. Xiao, P. Yakowek, X. Yan, Q. Ye, D. Yu, X. Zhao, J. Zhou, J.C. Medina, S.H. Olson, Rational design and binding mode duality of MDM2-p53 inhibitors, J. Med. Chem. 56 (2013) 40534070 . 